\title{
Calibration of Inductance Standards in the Maxwell-Wien Bridge Circuit*
}

\author{
Thomas L. Zapf \\ (February 28, 1961)
}

\begin{abstract}
This paper discusses the errors from residuals in the Maxwell-Wien bridge and the effect of these on the measurement of inductors in a bridge not having a Wagner ground. Particular attention is given to the use of substitution methods for accurate measurements and especially to the "equal-substitution" (comparison) method, which can yield excellent precision in the calibration of inductance standards.
\end{abstract}

\section{Introduction}

Self inductors and mutual inductors can be constructed to have inductance that is computable from their geometry and dimensions. Although the inductance of certain inductors having measurable mechanical dimensions and simple geometrical form can be computed with excellent accuracy, such inductors are not often used as reference standards for inductance measurements. In practice, inductance measurements are more conveniently made with reference to noncomputable reactances in the form of compact and stable reference standards of inductance or capacitance. Accurate values of inductance can be determined by comparing the reactance of the inductor with the reactance of either standard capacitors or standard inductors by use of appropriate bridge circuits. However, the most precise measurement of an unknown quantity (in this case inductance) is made by comparing it with a standard of like kind and magnitude, the small difference being measured by a corresponding small change in one element of the bridge circuit. When comparison methods are used, it is often possible to reduce the detrimental effects of residuals in the measuring circuit to such an extent that the precision of comparison is much better than the accuracy with which the calibration standard is known.

The Maxwell-Wien bridge circuit has long been used for the accurate measurement of inductance. For measurements of best accuracy with any alternating-current bridge circuit, even for comparison measurements, it is necessary to consider the effects of residuals, the ways of reducing these effects, and the handling of corrections to offset the net errors.

\section{Maxwell-Wien Bridge}

A bridge circuit originally developed by J. C. Maxwell [1] ${ }^{1}$ for use with ballistic detectors was adapted by M. Wien [2] for a-c measurements. For many years the circuit has been used widely by

*Contribution from the Radio Standards Laboratory, National Bureau of Standards, Boulder, Colo.

1 Figures in brackets indicate the literature references at the end of this paper. standardizing laboratories for measurements of inductance. Figure 1 shows a schematic of a wellshielded Maxwell-Wien bridge with Wagner arms for elimination of errors resulting from current diverted to ground through leakage impedances. The inductance, $L$, to be measured is placed in series with a resistance, $r_{L}$. If the components of the bridge arms shown in figure 1 were pure, the inductance measured when the bridge is balanced would be

$$
L=C R_{P} R_{S}
$$

and to obtain the balance it is necessary that

$$
\frac{R}{R_{S}}=\frac{R_{P}}{r_{L}}
$$

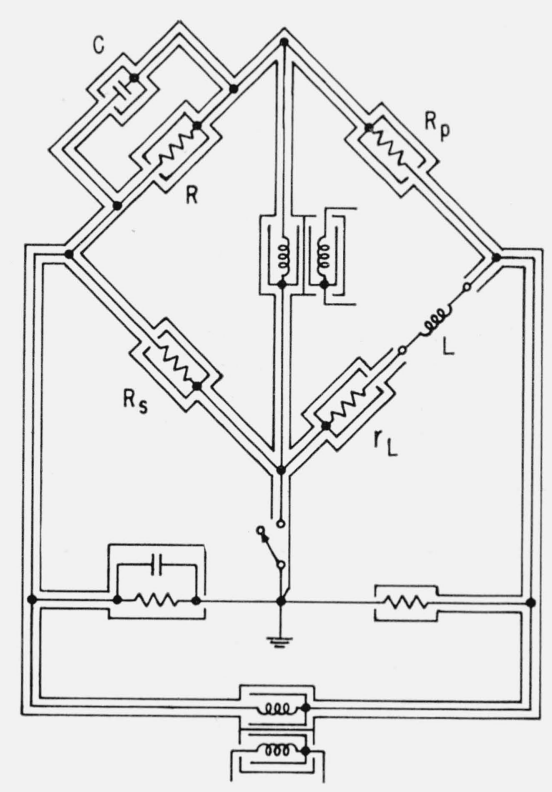

FIGURE 1. The Maxwell-Wien bridge circuit with complete shielding and a Wagner ground. 
In practical situations it is not possible to obtain completely pure components. The residuals in the components contribute to the systematic errors, and it is necessary to analyze the circuit sufficiently to correct for these errors or to determine the maximum uncertainty in the results if the errors are neglected. Figure 2 shows the components of figure 1 with the addition of appropriate residual components, but without the Wagner arms. For example, the series resistor, $r_{L}$ of figure 1 , in the inductance arm is represented in figure 2 by a pure resistance, $r_{L}$, in series with an inductance, $l_{L}$. The inductance, $l_{L}$, is a lumped constant that may be either positive or negative depending upon the relative magnitudes of inductive and capacitive effects. Although lumped constant residuals are not independent of frequency, the lumped constant concept provides a useful equivalent circuit at low frequencies. The other resistive components are similarly represented by a pure resistance and an effective series inductance. The variable capacitance, $C$, is presumed to contain an effective series resistance, $r$. The resistance $R_{L}$ in figure 2 is the equivalent series resistance of the inductor.

\section{Equation of Balance}

The impedances of the four bridge arms are

$$
\begin{gathered}
Z_{R G}=\frac{\left[(R+j \omega l)\left(1+\omega^{2} r^{2} C^{2}\right)\right.}{1+\omega^{2} r^{2} C^{2}+\omega C(\omega r C+j)(R+j \omega l)}, \\
Z_{P}=R_{P}+j \omega l_{P}, \\
Z_{L}=R_{L}+r_{L}+j \omega\left(L+l_{L}\right),
\end{gathered}
$$

and

$$
Z_{S}=R_{S}+j \omega l_{S}
$$

The derivation of eq (3) is given in the appendix. When the bridge is balanced

$$
Z_{R C} Z_{L}=Z_{P} Z_{S}
$$

After the multiplication indicated by eq (7) is performed, the equation of imaginary components can be written

$$
\begin{aligned}
\left(1+\omega^{2} r^{2} C^{2}\right) & {\left[R\left(L+l_{L}\right)+\left(R_{L}+r_{L}\right) l-R_{S} l_{P}-R_{P} l_{S}\right] } \\
=C & {\left[R R_{P} R_{S}+\omega\left(R R_{P} l_{S}+R R_{S} l_{P}+R_{P} R_{S} l\right) \omega r C\right.} \\
& \left.-\omega^{2}\left(R l_{P} l_{S}+R_{P} l l_{S}+R_{S} l_{P}\right)-\omega^{3} l l_{P} l_{S} \omega r C\right]
\end{aligned}
$$

Considerable simplification of eq (8) is possible if the residuals are expressed as time constants and dissipation factors, as follows. Let

$$
\begin{gathered}
\omega r C=D_{C}, \\
\frac{l}{R}=\tau_{R},
\end{gathered}
$$

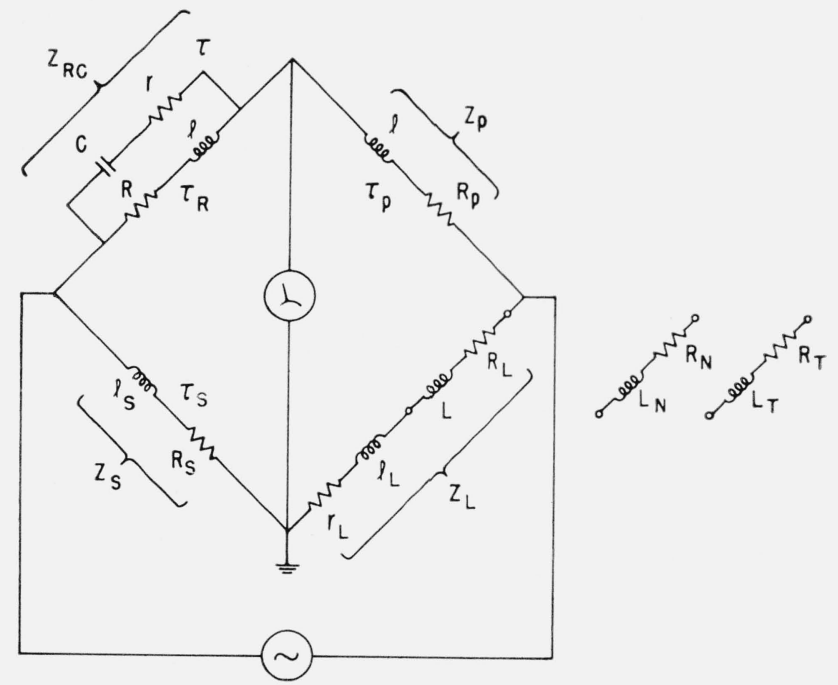

Figure 2. The Maxwell-Wien bridge circuit showing the residuals in the arms as lumped constants.

$$
\frac{l_{P}}{R_{P}}=\tau_{P}
$$

and

$$
\frac{l_{S}}{R_{S}}=\tau_{S}
$$

With negligible error it will be found that

$$
\begin{aligned}
L=C R_{P} R_{S}[ & 1-\omega^{2}\left(\tau_{P} \tau_{S}+\tau_{R} \tau_{P}+\tau_{R} \tau_{S}\right)-D_{C}^{2} \\
+ & \left.\omega\left(\tau_{P}+\tau_{S}+\tau_{R}\right) D_{C}-\omega^{3} \tau_{P} \tau_{S} \tau_{R} D_{C}\right] \\
& \quad-\left(R_{L}+r_{L}\right) \tau_{R}+\frac{R_{P} R_{S}}{R}\left(\tau_{P}+\tau_{S}\right)-l_{L}
\end{aligned}
$$

and a convenient simplification results if the small terms in brackets are replaced by the symbol $K$. Then the equation of balance becomes

$L=C R_{P} R_{S}(1+K)-\left(R_{L}+r_{L}\right) \tau_{R}+\frac{R_{P} R_{S}}{R}\left(\tau_{P}+\tau_{S}\right)-l_{L}$,

where

$$
\begin{aligned}
K=-\omega^{2}\left(\tau_{P} \tau_{S}+\right. & \left.\tau_{R} \tau_{P}+\tau_{R} \tau_{S}\right)-D_{C}^{2} \\
& +\omega\left(\tau_{P}+\tau_{S}+\tau_{R}\right) D_{C}-\omega^{3} \tau_{P} \tau_{S} \tau_{R} D_{C} .
\end{aligned}
$$

\section{Effect of Stray Capacitance}

Before analyzing this equation in detail it is profitable to consider the effect of stray capacitance across the terminals of the inductor. Such capacitance can be introduced by the leads connecting the inductor to the bridge (see fig. 3). For this analysis, whatever capacitance exists within the case of the inductor is considered not a part of $C_{S}$, but rather part of the inductor. It should be understood that this internal shunt capacitance contributes to the 


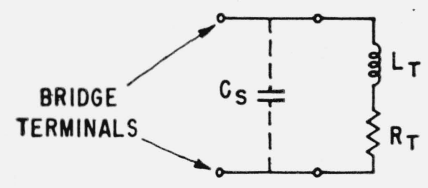

(a)

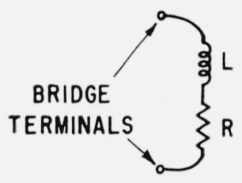

(b)
Figure 3. The inductor under test, $\mathrm{L}_{\mathrm{T}}$, is connected to bridge terminals with leads having stray capacitance, $\mathrm{C}_{\mathrm{S}}$, between them.

The bridge measures the equivalent inductance, $L$, which differs from $L_{T}$ because of the stray capacitance.

effective series inductance measured at the terminals of the inductor, thereby causing the effective series inductance to vary with frequency to some extent.

Figure 3 a shows the equivalent series inductance under test, $L_{T}$, in series with the internal resistance of the inductor, $R_{T}$. The stray capacitance, $C_{S}$, external to the inductor is in parallel with $L_{T}$ and $R_{T}$. The bridge measurement does not completely separate these elements but indicates the effective inductance, $L$, as shown in figure $3 \mathrm{~b}$. The effective series inductance, $L_{T}$, at the terminals of the inductor is related to the measured value, $L$, at the ends of the leads by the approximation

$$
L_{T} \approx L\left(1-\omega^{2} L_{T} C_{S}+\frac{R_{T}^{2} C_{S}}{L_{T}}+\omega^{2} R_{T}^{2} C_{S}^{2}\right) .
$$

The derivation of eq (16) is given in the appendix. The last three terms in parentheses constitute the small correction terms resulting from stray capacitance. The first and the last of these vary as the square of the frequency. The last term is usually much the smaller of the two in practical situations. The second correction term is not dependent upon frequency and must never be overlooked as a source of error in measurements of the highest accuracy. For example, in the measurement of a $10 \mathrm{~h}$ inductor having an internal resistance of $10,000 \mathrm{ohms}$, at the end of a coaxial cable having a capacitance of $100 \mathrm{pf}$, the error from neglecting the second correction term is $0.01 \mathrm{~h}$, equivalent to 0.1 percent of the quantity measured. Often the first correction term is significant while the other terms are negligible, and the effective series inductance then can be written

$$
L_{T} \approx L\left(1-\omega^{2} L_{T} C_{S}\right)
$$

However, in this paper both the first and second correction terms will be carried in the analysis to follow.

\section{Substitution Methods}

The existence of the residuals and the consequent correction terms in eqs (14) and (16) are detrimental to good accuracy for residuals are often very difficult to measure or estimate to better than an order of magnitude. To eliminate the effect on measurements of some of these residuals, substitution methods are used. For example, if a standard inductor of equal nominal value is substituted for the test inductor and the difference of inductance is measured by changing $C$ and $r_{L}$ (or $R$ ) slightly, then the residuals in the resistance arms of the bridge have negligible effect on the measurement. Also, the effect of the stray capacitance across the inductor under test or across the standard inductor is much reduced. The standard inductor must be very accurately known, however.

If accurate measurements of large inductors are contemplated and a shorting plug of negligible, or relatively small, inductance is substituted for the test inductor, $C_{S}$ must be reduced to a negligible value by complete shielding, and a Wagner ground should be used. The effect of the residual, $l_{L}$, in the resistor, $r_{L}$, is eliminated by this method only if $l_{L}$ is the same for the two conditions of balance.

The latter method, in which a relatively small inductance replaces the inductor under test, can be designated the "zero-substitution" method to distinguish it from the "equal-substitution" method, in which the test inductor is replaced by a standard of equal nominal inductance.

A general formula for inductance measurements by substitution methods with the Maxwell-Wien bridge may be derived from the two balance equations, the first with the inductor, $L_{T}$, in the circuit, and the second with a standard, $L_{N}$, in its place. If the small correction terms represented by $K$ in eq (14) are constant for both conditions of balance and if $C_{S}$ is changed only slightly (to $C_{S N}$ ) when the standard inductor or shorting plug is connected, then with certain justifiable approximations

$$
\begin{aligned}
& L_{T}=L_{N}+\left(C-C_{N}\right) R_{P} R_{S}\left[1-\omega^{2}\left(L_{T}+L_{N}\right) C_{S}+K\right] \\
&-\omega^{2} L_{T} L_{N}\left(C_{S}-C_{S N}\right) \\
& \quad+\left(R_{T}^{2}-R_{N}^{2}\right) C_{S}-\left(l_{L}-l_{L N}\right) .
\end{aligned}
$$

The derivation of eq (18) is given in the appendix. This equation is useful in showing the effect of uncertainties in the magnitude of circuit components on the determination of inductance.

If good quality components are used and the bridge is well designed, the time constants that contribute to $K$ will be small. However, $K$ cannot be determined with certainty and is somewhat variable (dependent upon actual circuit constants). The uncertainty in $K$ sets an ultimate practical limit to the accuracy of measurement of inductance by the Maxwell-Wien bridge.

\section{Zero-Substitution Method}

When the zero-substitution method is employed the small inductance, $L_{N}$, need not be accurately known, but the capacitor, $C$, and the resistance product, $R_{P} R_{S}$, must be known with better accuracy than that desired for the inductance, $L_{T}$. It is possible to construct very small inductances that are computable from geometry and dimensions. A shorting bar between two terminals has a finite inductance 
that can often be calculated or estimated well enough to serve as the "zero-reference", $L_{N}$, for bridge measurements of inductors of much larger magnitude. If the measured inductance is defined as the increase in inductance when a shorting-link or switch associated with the inductor is manipulated in a specified manner, then the actual inductance of the short circuit, $L_{N}$, is immaterial. The term $\left(l_{L}-l_{L N}\right)$ can be minimized if the resistance, $r_{L}$, is inductance compensated; i.e., if designed so that the effective series inductance remains constant as the resistance is varied. The uncertainties in this term may limit the accuracy of measurement of small inductors. If $C_{S}$ is not extremely small, it is evident that the uncertainty in $C_{S}$ can have a significant effect on the accuracy of measurement by this method. In eq (18) the term in brackets containing $C_{S}$ can become objectionably large when large values of inductance are being measured. For example, if the inductance being measured is approximately $10 \mathrm{~h}$, if $C_{S}=100 \mathrm{pf} \pm 10 \mathrm{pf}$, and if $\omega=10,000$ radians per second, then $\omega^{2} L C_{S}=0.10 \pm 0.01$. Thus the correction term is 10 percent of the measured inductance, and the measurement uncertainty is 1 percent from this cause alone, disregarding the probable lack of validity of the assumptions (when the correction terms are so large) that were used to derive eq (18). The effect of capacitance $C_{S}$ thus places a definite limitation on the accuracy which can be obtained by the zerosubstitution method with a grounded bridge. In order to obtain better accuracy when $\omega^{2} L$ is large it is necessary to use the "equal-substitution method" (described below) or to resort to separate shielding on the inductor leads with an ungrounded bridge using Wagner arms. This latter possibility involves considerations beyond the scope of this paper.

\section{Equal-Substitution Method}

If an accurately known standard inductance, $L_{N}$, is substituted for the test inductor, $L_{T}$, the capacitance, $C$, and the resistance product, $R_{P} R_{S}$, need not be so accurately calibrated. Between the two conditions of balance the only circuit components changed are $C$ and $r_{L}$, both by small amounts. The residuals $D_{C}$ and $l_{L}$ are essentially unchanged in this case, and all the other residuals are identical for both conditions of balance. For the equal-substitution method the general eq (18) can be rewritten with negligible additional error as

$$
\begin{aligned}
L_{T}= & L_{N}+\left(C-C_{N}\right) R_{P} R_{S}\left(1-2 \omega^{2} L_{T} C_{S}+K\right) \\
& -\omega^{2} L_{T}^{2}\left(C_{S}-C_{S N}\right)+\left(R_{T}^{2}-R_{N}^{2}\right) C_{S}-\left(l_{L}-l_{L N}\right) .
\end{aligned}
$$

In eqs (18) and (19) the stray capacitances, $C_{S}$ and $C_{S N}$, are not considered to be exactly equal; however, it is expected that they will be maintained nearly equal. The importance of this, even in equalsubstitution measurements, is evident from eq (19). In the comparison of $10 \mathrm{~h}$ inductors at an angular frequency of 10,000 radians per second, if the difference $C_{S}-C_{S N}$ is only $1 \mathrm{pf}$, then $\omega^{2} L_{T}^{2}\left(C_{S}-C_{S N}\right)$ is $0.01 \mathrm{~h}$. This error of 0.1 percent shows the impor- tance of maintaining $C_{S}$ nearly constant during the substitution.

The term $\left(l_{L}-l_{L N}\right)$ can be reduced to a minimum by employing an adjustable resistor, $r_{L}$, having inductance compensation. Uncertainties in this term may limit accuracy if the resistances of the test and standard inductors differ greatly, and the inductance compensation of the resistor, $r_{L}$, is inadequate. The adjustable resistor is in series with the inductor and is connected to the grounded corner of the bridge as shown in figure 2. If, instead, this resistor were connected to the ungrounded end of the inductance arm of the bridge, the capacitance to ground from the several decades of this resistor would shunt the inductor, and as the resistance is varied to accommodate inductors having different internal resistance the change in stray capacitance would cause an error that would be difficult to correct. If one end of the resistor is grounded, and if care is taken to keep the resistance as low as possible, the capacitance to ground within the resistor merely shunts the resistance, contributing to the effective residual inductance, $l_{L}$, which is generally insignificant.

The term $\left(\mathrm{R}_{T}^{2}-R_{N}^{2}\right) C_{S}$ deserves special attention because it is directly dependent upon the effective series resistances of the inductors. As an example of the effect of this term, consider that, in the measurement of a $10 \mathrm{~h}$ inductor, $R_{T}=10,000 \mathrm{ohms}$, $R_{N}=8,000 \mathrm{ohms}$, and $C_{S}=100 \mathrm{pf}$. Then the term $\left(R_{T}^{2}-R_{N}^{2}\right) C_{S}=0.0036 \mathrm{~h}$, nearly 0.04 percent of the quantity measured. This correction, being independent of frequency (if skin effect may be neglected) exists even at low frequencies, at which most of the other correction terms are negligible.

If the time constants, $\tau_{P}, \tau_{S}$, and $\tau_{R}$, are $1 \mu \mathrm{sec}$ or less and $D_{C}$ is 0.001 or less (these are conservative but reasonable estimates for commercially available components), and the angular frequency is 10,000 radians per second, the magnitude of $K$ is less than 0.0003. For a 1 percent difference in inductors, if this term were neglected the resulting error would be only $3 \mathrm{ppm}$ of the measured inductance.

Thus, the equal-substitution method can be employed without the necessity of using a Wagner ground providing care is taken to keep the stray capacitance, $C_{S}$, reasonably small and nearly constant and to apply corrections to the measured values to offset errors resulting from the difference in the internal resistances of the inductors.

\section{Effect of Frequency Difference}

It has been assumed that the frequency of the a-c supply is stable. If the frequency, $\omega_{N}$, when the standard inductor is in the circuit is not equal to the frequency, $\omega$, when the test inductor is connected, there can be an error resulting from the stray capacitance, $C_{S}$. A difference of frequency between the test balance and the standard balance will necessitate the addition of the term

$$
-C_{S} L_{T} L_{N}\left(\omega^{2}-\omega_{N}^{2}\right)
$$


to the right side of eq (18), and since $\omega \approx \omega_{N}$ in any practical case, this can be factored to produce

$$
-2 \omega C_{S} L_{T} L_{N}\left(\omega-\omega_{N}\right) .
$$

Since $L_{T} \approx L_{N}$ for the equal-substitution method, the additional term to be added to eq (19) is

$$
-2 \omega C_{S} L_{T}^{2}\left(\omega-\omega_{N}\right)
$$

If $\omega=10,000$ radians per second, $\omega_{N}=10,010$ radians per second (a $0.1 \%$ difference), and $C_{S}=100$ $\mathrm{pf}$, for the measurement of a $10 \mathrm{~h}$ inductor by the equal-substitution method the magnitude of the correction term is $0.002 \mathrm{~h}$, an error of 0.02 percent. It is important to realize that this analysis of the effect of frequency variation does not account for the change of effective series inductance with frequency resulting from eddy currents, skin effect, or distributed capacitance within the inductors.

\section{Calibration of Inductors}

In recent years standard inductors having good stability have become available in a wide range of nominal values. These have made accurate measurements by the equal-substitution method feasible and convenient. Several complete sets of standards are maintained in the laboratories of NBS both at Washington and at Boulder, and are used regularly for calibrating similar standards submitted for certification. The values assigned to NBS working standards ultimately depend upon a computable inductor or capacitor.

The equal-substitution method can be utilized with a variety of a-c bridge circuits. The essential requirements are that the bridge have good shorttime stability, adequate resolution, and means for externally equalizing the storage factor $(Q)$ of the inductors being compared. An advantage of the equal-substitution method is the lessened need for accuracy of adjustment of the bridge components because the bridge is used merely to measure small differences. A number of commercially available bridges embody the Maxwell-Wien bridge circuit to which the analysis given in this paper is primarily devoted. It is almost invariably necessary, however, to improve their resolution by adding a calibrated variable capacitor in parallel with those built into the bridge.

The resistor, $r_{L}$, is partially inductance compensated and is connected in series with the cable connecting the inductors to the bridge. It has been determined that the existing variations of inductance are negligible relative to the inductance being measured by this method. This resistor may be regarded as serving the purpose of externally equalizing the $Q$ of the standard and test inductors.

It is seldom necessary to apply a correction for the term $\left(R_{T}^{2}-R_{N}^{2}\right) C_{S}$ in eq (19). On rare occasions when this term is significant, a crude measurement of $R_{T}$ and $R_{N}$ is adequate.
The resistance product (range) in the bridge is set so that the difference between the test and the standard inductors can be accommodated by adjustment of the externally connected variable capacitor and resistor, the other bridge controls being left unchanged.

The analysis of errors in the Maxwell-Wien bridge circuit described in this paper was carried out as part of the investigation of the feasibility of adopting the equal-substitution method for the rapid and convenient measurement of inductance.

The use of the equal-substitution method for the precise comparison of inductors at the Electronic Calibration Center, NBS, Boulder, Colo., was instigated by Chester Peterson, NBS, Washington, D.C. The author is indebted to Mr. Peterson for helpful comments and suggestions pertaining to this paper.

\section{Appendix: Derivation of Equations}

Equation (3) is derived from the equation of the impedance of $Z_{C}$ and $Z_{R}$ in parallel.

$$
\begin{aligned}
\frac{1}{Z_{R C}} & =\frac{1}{R+j \omega l}+\frac{\omega C}{\omega r C-j} \\
& =\frac{1}{R+j \omega l}+\frac{\omega C(\omega r C+j)}{\omega^{2} r^{2} C^{2}+1} \\
& =\frac{1+\omega^{2} r^{2} C^{2}+\omega C(\omega r C+j)(R+j \omega l)}{(R+j \omega l)\left(1+\omega^{2} r^{2} C^{2}\right)} .
\end{aligned}
$$

Therefore

$$
Z_{R C}=\frac{(R+j \omega l)\left(1+\omega^{2} r^{2} C^{2}\right)}{1+\omega^{2} r^{2} C^{2}+\omega C(\omega r C+j)(R+j \omega l)} .
$$

Equation (8) is obtained by substituting eqs (3), (4), (5), and (6) into eq (7), giving

$$
\begin{aligned}
&\left(1+\omega^{2} r^{2} C^{2}\right)(R+j \omega l)\left[\left(R_{L}+r_{L}\right)+j \omega\left(L+l_{L}\right)\right] \\
&=\left[1+\omega^{2} r^{2} C^{2}+\omega C(\omega r C+j)(R+j \omega l)\right] \\
& \quad\left(R_{P}+j \omega l_{P}\right)\left(R_{S}+j \omega l_{S}\right),
\end{aligned}
$$

from which the equation of imaginary components is

$$
\begin{aligned}
& \left(1+\omega^{2} r^{2} C^{2}\right)\left[R\left(L+l_{L}\right)+\left(R_{L}+r_{L}\right) l-R_{S} l_{P}-R_{P} l_{S}\right] \\
& =C\left[R R_{P} R_{S}+\omega\left(R R_{P} l_{S}+R R_{S} l_{P}+R_{P} R_{S} l\right) \omega r C\right. \\
& \left.\quad-\omega^{2}\left(R l_{P} l_{S}+R_{P} l l_{S}+R_{S} l l_{P}\right)-\omega^{3} l l_{P} l_{S} \omega r C\right] .
\end{aligned}
$$

Equation (16) describes the effect of stray capacitance. Figure 3 a represents an inductor, $L_{T}$, having an internal resistance, $R_{T}$, connected to the bridge terminals by a cable having shunt capacitance, $C_{S}$. 
The impedance of this circuit, $Z$, is derived as follows

$$
\begin{aligned}
\frac{1}{Z} & =\frac{1}{R_{T}+j \omega L_{T}}+j \omega C_{S}, \\
Z & =\frac{\left(R_{T}+j \omega L_{T}\right)\left(1-\omega^{2} L_{T} C_{S}-j \omega R_{T} C_{S}\right)}{\left(1-\omega^{2} L_{T} C_{S}+j \omega R_{T} C_{S}\right)\left(1-\omega^{2} L_{T} C_{S}-j \omega R_{T} C_{S}\right)}, \\
Z & =\frac{R_{T}+j \omega\left[L_{T}\left(1-\omega^{2} L_{T} C_{S}\right)-R_{T}^{2} C_{S}\right]}{\left(1-\omega^{2} L_{T} C_{S}\right)^{2}+\omega^{2} R_{T}^{2} C_{S}^{2}} .
\end{aligned}
$$

In the equivalent circuit shown in figure $3 \mathrm{~b}$

$$
Z=R+j \omega L,
$$

and from the two preceding equations it is evident that

$$
L=\frac{L_{T}\left(1-\omega^{2} L_{T} C_{S}\right)-R_{T}^{2} C_{S}}{\left(1-\omega^{2} L_{T} C_{S}\right)^{2}+\omega^{2} R_{T}^{2} C_{S}^{2}}
$$

and, with negligible error,

$$
L=\frac{L_{T}\left(1-\omega^{2} L_{T} C_{S}-\frac{R_{T}^{2} C_{S}}{L_{T}}\right)}{\left(1-2 \omega^{2} L_{T} C_{S}+\omega^{2} R_{T}^{2} C_{S}^{2}\right)} .
$$

It should be noted that the discarded term, $\omega^{4} L_{T}^{2} C_{S}^{2}$, in the denominator is always much smaller than the term, $2 \omega^{2} L_{T} C_{S}$, which is usually much smaller than 1 in practical inductance measurements at low audiofrequencies. Since the terms in parentheses, other than 1, are small, this equation can be solved for $L_{T}$ and simplified by neglecting higher order terms.

$$
L_{T} \approx L\left(1-\omega^{2} L_{T} C_{S}+\frac{R_{T}^{2} C_{S}}{L_{T}}+\omega^{2} R_{T}^{2} C_{S}^{2}\right) .
$$

Equation (18) is obtained from the following two equations which are derived from eq (14) modified according to eq (16)

$$
\begin{aligned}
L_{T}=C R_{P} R_{S}\left(1-\omega^{2} L_{T} C_{S}+\frac{R_{T}^{2} C_{S}}{L_{T}}\right)(1+K) \\
-\left(R_{L}+r_{L}\right) \tau_{R}+\frac{R_{P} R_{S}}{R}\left(\tau_{P}+\tau_{S}\right)-l_{L}
\end{aligned}
$$

and

$$
\begin{aligned}
L_{N}=C_{N} R_{P} R_{S}( & \left.1-\omega^{2} L_{N} C_{S N}+\frac{R_{N}^{2} C_{S N}}{L_{N}}\right)(1+K) \\
& \quad-\left(R_{L N}+r_{L N}\right) \tau_{R}+\frac{R_{P} R_{S}}{R}\left(\tau_{P}+\tau_{S}\right)-l_{L N} .
\end{aligned}
$$

In eq (15) it will be noted that at the higher frequencies the first term in $K$ is the largest term, and that at lower frequencies all terms in $K$ are small and often negligible. The first term in $K$ is independent of $D_{C}$, which may vary slightly between the two conditions of balance; hence, the assumption that $K$ is constant is justifiable. In the above equations since

and

$$
C R_{P} R_{S} \approx L_{T}
$$

and

$$
C_{N} R_{P} R_{S} \approx L_{N}
$$

$$
\left(R_{L}+r_{L}\right) \approx\left(R_{L N}+r_{L N}\right)
$$

$C R_{P} R_{S} R_{T}^{2} C_{S} / L_{T} \approx R_{T}^{2} C_{S}$ and

$$
C_{N} R_{P} R_{S} R_{N}^{2} C_{S N} / L_{N} \approx R_{N}^{2} C_{S N} .
$$

Therefore,

$$
\begin{aligned}
L_{T}-L_{N} & =\left[\left(C-C_{N}\right) R_{P} R_{S}-R_{P} R_{S} \omega^{2}\left(C L_{T} C_{S}\right.\right. \\
& \left.\left.-C_{N} L_{N} C_{S N}\right)+R_{T}^{2} C_{S}-R_{N}^{2} C_{S N}\right](1+K)-\left(l_{L}-l_{L N}\right) .
\end{aligned}
$$

The factor $\left(C L_{T} C_{S}-C_{N} L_{N} C_{S N}\right)$ can be expanded as follows:

$$
\begin{aligned}
\left(C L_{T} C_{S}-C_{N} L_{N} C_{S N}\right)=\left(C-C_{N}\right)( & \left.L_{T} C_{S}+L_{N} C_{S N}\right) \\
& +C_{N} L_{T} C_{S}-C L_{N} C_{S N} .
\end{aligned}
$$

If $C_{S}$ and $C_{S N}$ can be reduced to zero, this factor becomes zero, and the equation is greatly simplified. However, if $C_{S}$ is not zero, but if $C_{S}$ approximately equals $C_{S N}$,

$$
\begin{aligned}
C L_{T} C_{S}-C_{N} L_{N} C_{S N}=\left(C-C_{N}\right)\left(L_{T}\right. & \left.+L_{N}\right) C_{S} \\
& +C_{N} L_{T} C_{S}-C L_{N} C_{S N}
\end{aligned}
$$

and

$$
\begin{aligned}
L_{T}-L_{N}=\{ & \left(C-C_{N}\right) R_{P} R_{S}\left[1-\omega^{2}\left(L_{T}+L_{N}\right) C_{S}\right] \\
& \quad-R_{P} R_{S} \omega^{2}\left(C_{N} L_{T} C_{S}-C L_{N} C_{S N}\right) \\
& \left.+\left(R_{T}^{2}-R_{N}^{2}\right) C_{S}\right\}(1+K)-\left(l_{L}-l_{L N}\right),
\end{aligned}
$$

and with negligible error the substitution $C_{N} R_{P} R_{S}$ $=L_{N}$ and $C R_{P} R_{S}=L_{T}$ can be made in the small correction terms. The second term in braces can be reduced to $-\omega^{2} L_{T} L_{N}\left(C_{S}-C_{S N}\right)$. If the terms under consideration are not relatively small, the approximations are not valid.

Neglecting second order terms, the above equation can be simplified, giving eq (18):

$$
\begin{aligned}
L_{T}=L_{N} & +\left(C-C_{N}\right) R_{P} R_{S}\left[1-\omega^{2}\left(L_{T}+L_{N}\right) C_{S}+K\right] \\
& -\omega^{2} L_{T} L_{N}\left(C_{S}-C_{S N}\right)+\left(R_{T}^{2}-R_{N}^{2}\right) C_{S}-\left(l_{L}-l_{L N}\right) .
\end{aligned}
$$

\section{References}

[1] J. C. Maxwell, A treatise on electricity and magnetism, 1st ed., 2, 377-379 (1873), or 3d ed., Art. 778.

[2] Max Wien, Ann. der Phys., 44, 689-712 (1891).

[3] H. L. Curtis, Electrical Measurements, 113-117, McGrawHill Book Co., Inc., New York, N.Y. (1937).

(Paper 65C3-69) 